\title{
High/low-moment phase transition in hexagonal Mn-Fe-P-Si compounds
}

\author{
N. H. Dung,,${ }^{1,}$ L. Zhang,,${ }^{1,2}$ Z. Q. Ou, ${ }^{1}$ L. Zhao, ${ }^{3}$ L. van Eijck, ${ }^{4}$ A. M. Mulders, ${ }^{5}$ M. Avdeev, ${ }^{6}$ E. Suard, ${ }^{7}$ \\ N. H. van Dijk, ${ }^{1}$ and Ekkes Brück ${ }^{1}$ \\ ${ }^{1}$ Fundamental Aspects of Materials and Energy, Faculty of Applied Sciences, Delft University of Technology, Mekelweg 15 , \\ 2629 JB Delft, Netherlands \\ ${ }^{2}$ BASF Nederland B.V., Strijkviertel 67, 3454 PK De Meern, Netherlands \\ ${ }^{3}$ Department of Materials Science and Engineering, Delft University of Technology, Mekelweg 2, 2628 CD Delft, Netherlands \\ ${ }^{4}$ Neutron and Positron Methods in Materials, Faculty of Applied Sciences, Delft University of Technology, Mekelweg 15, \\ 2629 JB Delft, Netherlands \\ ${ }^{5}$ The University of New South Wales in Canberra, Canberra, Australian Capital Territory 2600, Australia \\ ${ }^{6}$ Bragg Institute, Australian Nuclear Science and Technology Organization (ANSTO), Kirrawee DC NSW 2232, Australia \\ ${ }^{7}$ Institut Laue Langevin (ILL), F-38042 Grenoble, France \\ (Received 23 September 2011; published 27 July 2012)
}

\begin{abstract}
Using high-resolution neutron diffraction measurements for Mn-rich hexagonal Mn-Fe-P-Si compounds, we show that the substitution of $\mathrm{Mn}$ for $\mathrm{Fe}$ on the $3 f$ sites results in a linear decrease of the $\mathrm{Fe} / \mathrm{Mn}(3 f)$ magnetic moments, while the $\operatorname{Mn}(3 g)$ magnetic moments remain constant. With increasing temperature, the $\mathrm{Mn}(3 g)$ magnetic moments show almost no change, while the $\mathrm{Fe} / \mathrm{Mn}(3 f)$ moments decrease quickly when the transition temperature is approached. The reduction of the magnetic moments at the transition temperature and in the high-temperature range is discussed based on changes in interatomic distances and lattice parameters and high-temperature magnetic-susceptibility measurement.
\end{abstract}

DOI: 10.1103/PhysRevB.86.045134

PACS number(s): 75.30.Sg, 64.70.kd, 75.30.Kz, 75.50.Bb

\section{INTRODUCTION}

The interplay between crystal structure and magnetism, which is often very pronounced in first-order magnetic transition (FOMT) materials, is proposed to be at the core of giant magnetocaloric effect (MCE). Up to now, giant MCE has been discovered in several materials which are promising for room-temperature magnetic refrigeration applications. ${ }^{1-7}$ Although a magnetic phase transition coupled with a change in crystal structure can be observed clearly in these materials, pinpointing the microscopic mechanism for the coupling between magnetism and crystal structure is not easy. By using X-ray magnetic circular dichroism and density functional theory, the mechanism of first-order bond-breaking magnetostructural transition in $\mathrm{Gd}_{5} \mathrm{Ge}_{4-x} \mathrm{Si}_{x}$ was clarified with emphasis on the role of Ge in bridging ferromagnetism. ${ }^{8}$ Spindependent hybridization between $\mathrm{Ge} 4 p$ and $\mathrm{Gd} 5 d$ conduction states enables long-range Ruderman-Kittel-Kasuya-Yosida (RKKY) ferromagnetic interactions between Gd $4 f$ moments in adjacent $\mathrm{Gd}$ slabs connected by $\mathrm{Ge} / \mathrm{Si}-\mathrm{Ge} / \mathrm{Si}$ bonds. Hence, ferromagnetic order will be destroyed if the RKKY interactions are weakened above the $\mathrm{Ge} / \mathrm{Si}-\mathrm{Ge} / \mathrm{Si}$ bond-breaking transition. The chemical bonding between $\mathrm{Ge} / \mathrm{Si}$ atoms plays an important role in first-order magneto-structural transition for this class of materials. For $\mathrm{LaFe}_{13-x} \mathrm{Si}_{x}$, electronic structure calculations indicated the existence of several shallow minima and maxima in the total energy vs the spin-moment curve, resulting in a ferro- to paramagnetic transition that involves a series of consecutive transitions. ${ }^{9}$ The extremes in the theoretical total energy profile are very sensitive to the lattice parameter of the cubic $\mathrm{LaFe}_{13-x} \mathrm{Si}_{x}$ phase. Although a peculiar series of consecutive field-induced transitions were realized in $\mathrm{La}(\mathrm{Fe}, \mathrm{Si})_{13} \mathrm{H}_{x}$ under pressure ${ }^{10}$ a microscopic mechanism is still lacking for the FOMT in this class of materials. Similarly, for $\mathrm{Ni}_{0.50} \mathrm{Mn}_{0.50-x} \mathrm{Sn}_{x}$ with an inverse MCE, the FOMT is often ascribed to a change in atomic distance at the martensitic transition that modifies magnetic properties of the alloy. ${ }^{5}$ However, ab initio electronic structure calculations still need to be done for a microscopic interpretation of the FOMT. ${ }^{5}$

First-principle electronic-structure calculations for hexagonal $\mathrm{MnFe}(\mathrm{P}, \mathrm{Si})$ revealed the coexistence of strong and weak magnetism in alternate atomic layers. ${ }^{7}$ These atomic layers are formed by the preferential occupation of $\mathrm{Mn}$ and $\mathrm{Fe}$ on the $3 g$ and $3 f$ sites, respectively. While the magnetic transition only causes a small reduction in the Mn moments on the $3 g$ sites, the disappearance of the Fe moments in the paramagnetic state demonstrates that there is a strong coupling between magnetism and crystal lattice. The competition between chemical bonding and moments is proposed to be at the basis of the FOMT. Therefore, a detailed study of the magnetic moments on different sites is needed to clarify the origin of the first-order magneto-elastic transition.

In this paper, we focus on the determination of the magnetic structure and magnetic moments using neutron diffraction measurements on Mn-rich hexagonal $\mathrm{Mn}_{x} \mathrm{Fe}_{1.95-x} \mathrm{P}_{0.50} \mathrm{Si}_{0.50}$ compounds $(x>1.0)$ with special attention paid to compounds $(x<1.4)$ displaying a first-order magneto-elastic transition. ${ }^{11}$ The reduction of the moment caused by the first-order magneto-elastic transition is discussed based on a sudden change in the interatomic distances at the transition temperature $\left(T_{\mathrm{C}}\right)$, an anomalous thermal expansion of the lattice parameters in the paramagnetic state and high-temperature magnetic-susceptibility measurement.

\section{EXPERIMENTAL DETAILS}

The Mn-rich $\mathrm{Mn}_{x} \mathrm{Fe}_{1.95-x} \mathrm{P}_{0.50} \mathrm{Si}_{0.50}$ compounds were prepared as described in Ref. 11. To make the samples more homogeneous, they were reannealed at $1373 \mathrm{~K}$ for $20 \mathrm{~h}$ before being 
quenched in cold water. A superconducting quantum interference device (SQUID) magnetometer (Quantum Design MPMS $5 \mathrm{XL}$ ) with the reciprocating sample option (RSO) mode was employed for magnetic measurements below $400 \mathrm{~K}$. Hightemperature magnetic measurements were carried out using a vibrating sample magnetometer (VSM; model LakeShore 7307) with a high-temperature oven (model 73034). The neutron diffraction data were collected at the Bragg Institute of the Australian Nuclear Science and Technology Organization (ANSTO) on the ECHIDNA high-resolution powder diffractometer ${ }^{12}$ with an incident wavelength of $1.622 \AA$ for the $x=1.20,1.25$, and 1.30 samples, and at the Institut Laue-Langevin (ILL) on the D2B high-resolution powder diffractometer ${ }^{13}$ with an incident wavelength of $1.595 \AA$ for the $x=1.95$ sample. The sample powder was contained in a vanadium can which is mounted in a cryostat. The measurements were carried out at fixed temperatures from 5 to $400 \mathrm{~K}$ in zero field. The $\mathrm{x}$-ray diffraction patterns were collected at various temperatures in zero field using a PANalytical X-pert Pro diffractometer equipped with an Anton Paar TTK450 low-temperature chamber using $\mathrm{Cu} \mathrm{K} \alpha$ radiation, a secondary-beam flat-crystal monochromator and a multichannel X'celerator detector. Starting at $150 \mathrm{~K}$, each Xray pattern was recorded at a constant temperature, and the following one was recorded at a higher temperature, up to $500 \mathrm{~K}$.

\section{RESULTS AND DISCUSSION}

All the samples studied display a para- to ferromagnetic transition. Figure 1(a) shows the neutron diffraction patterns for the $x=1.30$ sample at $285 \mathrm{~K}$ in the paramagnetic state and $5 \mathrm{~K}$ in the ferromagnetic state. The Rietveld refinement carried out using the FullProf program ${ }^{14}$ confirms hexagonal $\mathrm{Fe}_{2} \mathrm{P}$-type of structure (space group $P-62 m$ ). The results listed in Table I show that $\mathrm{Mn}$ prefers the $3 g$ sites, while Fe favors the $3 f$ sites. In the Mn-rich compounds, some Mn will occupy the $3 f$ sites (see Fig. 2). The refinement also indicates that $\mathrm{P}$ and $\mathrm{Si}$ are randomly distributed on the $2 c$ and $1 b$ sites. Earlier studies on $\mathrm{MnFe}(\mathrm{P}, \mathrm{Si})$ have pointed out that the magnetic moments prefer to be in the $a b$ plane rather than in the $c$ direction, as observed in $\mathrm{Fe}_{2} \mathrm{P}^{15,16}$ In the present study, the low-temperature diffraction pattern fits well when the magnetic moments are aligned in the $a b$ plane, indicating that replacing some Fe with Mn on the $3 f$ sites does not affect the preferential alignment of the magnetic moments.

Recently, we have shown that, for the Mn-rich $\mathrm{Mn}_{x} \mathrm{Fe}_{1.95-x} \mathrm{P}_{0.50} \mathrm{Si}_{0.50}$ compounds with increasing $\mathrm{Mn} / \mathrm{Fe}$ ratios, the first-order magneto-elastic transition $(x<1.40)$ changes into a first-order magneto-structural transition via a second-order isostructural magnetic transition $(x \geqslant 1.40){ }^{11}$ However, the hexagonal structure is stable in the ferromagnetic state at low temperature for all $\mathrm{Mn} / \mathrm{Fe}$ ratios. By using neutron diffraction, we find that the alignment of the magnetic moments in the $a b$ plane is also maintained until $\mathrm{Fe}$ is completely replaced by $\mathrm{Mn}$ on the $3 f$ sites [see Fig. 1(b) and Table I]. Interestingly, the $\mathrm{Mn}(3 g)$ moments remain constant at about $2.6 \mu_{\mathrm{B}}$, while the $\mathrm{Fe} / \mathrm{Mn}(3 f)$ moments decrease linearly from $1.5 \mu_{\mathrm{B}}$ for $x=1.20$ down to $0.4 \mu_{\mathrm{B}}$ for $x=1.95$ (see Fig. 3). The total moments obtained from macroscopic measurements of the low-temperature saturation
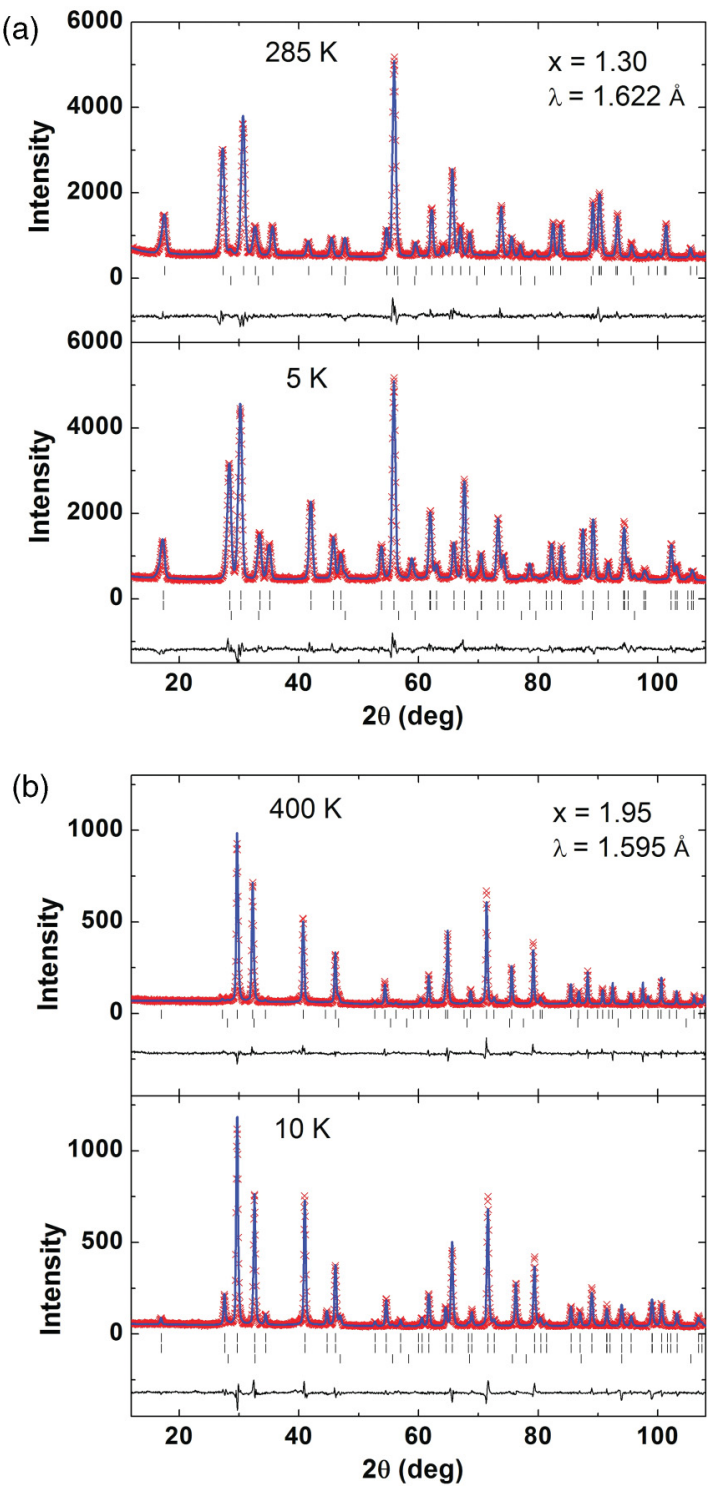

FIG. 1. (Color online) Observed (crosses), calculated (continuous lines) neutron diffraction patterns for hexagonal (a) $\mathrm{Mn}_{1.30} \mathrm{Fe}_{0.65} \mathrm{P}_{0.50} \mathrm{Si}_{0.50}\left(\lambda=1.622 \AA\right.$ ) and (b) $\mathrm{Mn}_{1.95} \mathrm{P}_{0.50} \mathrm{Si}_{0.50}$ $(\lambda=1.595 \AA)$ in the para- (upper plot) and ferromagnetic (lower plot) state. Differences are shown in the low part of the plots. The low-temperature diffraction patterns were fitted with the magnetic moments aligned in the $a b$ plane. The refined parameters are listed in Table I. A very small amount of cubic $(\mathrm{Mn}, \mathrm{Fe})_{3} \mathrm{Si}$ impurity phase is detected. Vertical lines indicate the diffraction peaks for the nuclear (top) and magnetic (middle, if any) structure of the main phase, and the impurity phase $(\mathrm{Mn}, \mathrm{Fe})_{3} \mathrm{Si}$ (bottom).

magnetization are very close to those derived from the neutron diffraction patterns at 3-10 K. The linear reduction of the $\mathrm{Fe} / \mathrm{Mn}(3 f)$ moments for increasing $x$ indicates that the $\mathrm{Mn}$ atom may develop a much lower magnetic moment than the $\mathrm{Fe}$ atom on the $3 f$ sites.

To investigate the thermal evolutions of the magnetic and structural properties, neutron diffraction measurements were performed at different temperatures for the $x=1.25$ sample exhibiting a first-order magneto-elastic transition. At $280 \mathrm{~K}$, coexistence of the ferro- and paramagnetic phases was detected 
TABLE I. Structural parameters of hexagonal $\mathrm{Mn}_{1.30} \mathrm{Fe}_{0.65} \mathrm{P}_{0.50} \mathrm{Si}_{0.50}$ and $\mathrm{Mn}_{1.95} \mathrm{P}_{0.50} \mathrm{Si}_{0.50}$ in the paramagnetic and ferromagnetic state. Group space: $P-62 m$. Atomic positions: $3 g\left(x_{1}, 0,1 / 2\right) ; 3 f\left(x_{2}, 0,0\right) ; 2 c(1 / 3,2 / 3,0)$; and $1 b(0,0,1 / 2)$. These parameters are derived from neutron diffraction patterns in Fig. 1.

\begin{tabular}{|c|c|c|c|c|c|}
\hline \multirow{2}{*}{\multicolumn{2}{|c|}{ Refined parameters }} & \multicolumn{2}{|c|}{$x=1.30$} & \multicolumn{2}{|c|}{$x=1.95$} \\
\hline & & $\begin{array}{c}285 \mathrm{~K} \\
\text { Paramagnetic }\end{array}$ & $\begin{array}{c}5 \mathrm{~K} \\
\text { Ferromagnetic }\end{array}$ & $\begin{array}{c}400 \mathrm{~K} \\
\text { Paramagnetic }\end{array}$ & $\begin{array}{c}10 \mathrm{~K} \\
\text { Ferromagnetic }\end{array}$ \\
\hline \multirow[t]{3}{*}{ Unit cell } & $a(\AA)$ & $6.11390(4)$ & $6.20611(4)$ & $6.21889(4)$ & $6.2145(1)$ \\
\hline & $c(\AA)$ & $3.42997(4)$ & $3.29842(4)$ & $3.38478(3)$ & $3.3400(1)$ \\
\hline & $V\left(\AA^{3}\right)$ & $111.034(2)$ & $110.021(2)$ & $113.367(1)$ & $111.709(4)$ \\
\hline \multirow[t]{4}{*}{$3 g$} & $x_{1}$ & $0.5918(3)$ & $0.5971(3)$ & $0.5948(3)$ & $0.5968(3)$ \\
\hline & $n(\mathrm{Mn}) / n(\mathrm{Fe})$ & $0.249 / 0.001(1)$ & $0.249 / 0.001(1)$ & $0.25 / 0$ & $0.25 / 0$ \\
\hline & $B\left(\AA^{2}\right)$ & $0.83(4)$ & $0.44(3)$ & $0.89(4)$ & $0.11(3)$ \\
\hline & $M\left(\mu_{\mathrm{B}}\right)$ & & $2.74(4)$ & & $2.61(3)$ \\
\hline \multirow[t]{4}{*}{$3 f$} & $x_{2}$ & $0.2562(2)$ & $0.2585(2)$ & $0.2531(4)$ & $0.2531(4)$ \\
\hline & $n(\mathrm{Fe}) / n(\mathrm{Mn})$ & $0.168 / 0.082(1)$ & $0.168 / 0.082(1)$ & $0 / 0.25$ & $0 / 0.25$ \\
\hline & $B\left(\AA^{2}\right)$ & $0.81(3)$ & $0.48(2)$ & $1.14(3)$ & $0.35(3)$ \\
\hline & $M\left(\mu_{\mathrm{B}}\right)$ & & $1.20(4)$ & & $0.36(3)$ \\
\hline \multirow[t]{2}{*}{$2 c$} & $n(\mathrm{P}) / n(\mathrm{Si})$ & $0.066 / 0.101(2)$ & $0.066 / 0.101(2)$ & $0.064 / 0.103(6)$ & $0.064 / 0.103(6)$ \\
\hline & $B\left(\AA^{2}\right)$ & $0.39(4)$ & $0.42(4)$ & $0.59(5)$ & $0.05(3)$ \\
\hline \multirow[t]{5}{*}{$1 b$} & $n(\mathrm{P}) / n(\mathrm{Si})$ & $0.059 / 0.024(2)$ & $0.059 / 0.024(2)$ & $0.061 / 0.022(6)$ & $0.061 / 0.022(6)$ \\
\hline & $B\left(\AA^{2}\right)$ & $0.85(6)$ & $0.53(4)$ & $1.21(8)$ & $0.30(4)$ \\
\hline & $R p(\%)$ & 3.75 & 4.27 & 5.61 & 6.19 \\
\hline & $w R p(\%)$ & 4.92 & 5.52 & 7.31 & 7.99 \\
\hline & $\chi^{2}$ & 3.42 & 4.16 & 4.15 & 4.99 \\
\hline
\end{tabular}

(see Fig. 4). The fraction of the ferro- to paramagnetic phase is estimated to be $33: 67$. While the $\operatorname{Mn}(3 g)$ moments show
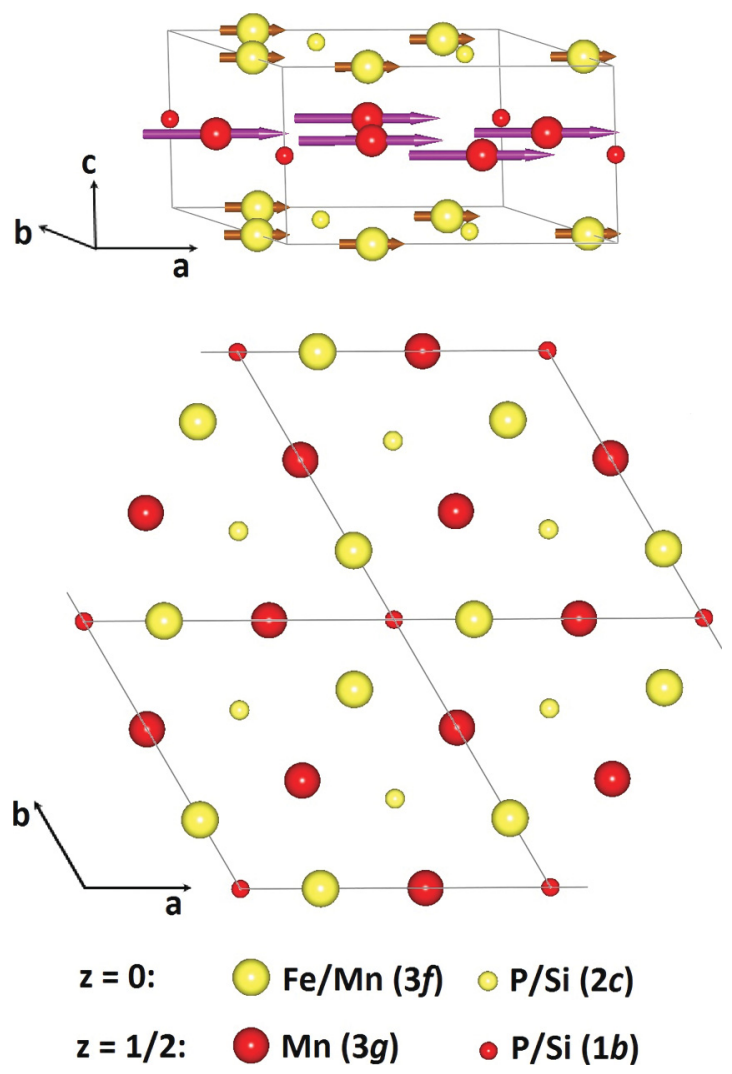

FIG. 2. (Color online) Atomic and spin arrangement within the unit cell of the Mn-rich $(\mathrm{Mn}, \mathrm{Fe})_{2}(\mathrm{P}, \mathrm{Si})$ compounds. almost no change for increasing temperature, the $\mathrm{Fe} / \mathrm{Mn}(3 f)$ moments rapidly decrease near $T_{\mathrm{C}}$ (see Fig. 5). This behavior is similar to that observed for $\mathrm{Mn}_{1.1} \mathrm{Fe}_{0.9} \mathrm{P}_{0.8} \mathrm{Ge}_{0.2}$ by Liu et al. ${ }^{17}$ This may be the signature for the reduction of magnetic moments, especially on the $3 f$ sites, at $T_{\mathrm{C}}$ which we proposed based on theoretical calculations. ${ }^{7}$

Figure 6 illustrates the temperature dependence of the lattice parameters and the distances between the magnetic atoms and the nearest neighbors for the $x=1.25$ sample. At temperatures below $T_{\mathrm{C}}$, the lattice parameters change in the opposite sense, i.e. the $a$ parameter decreases, while the $c$

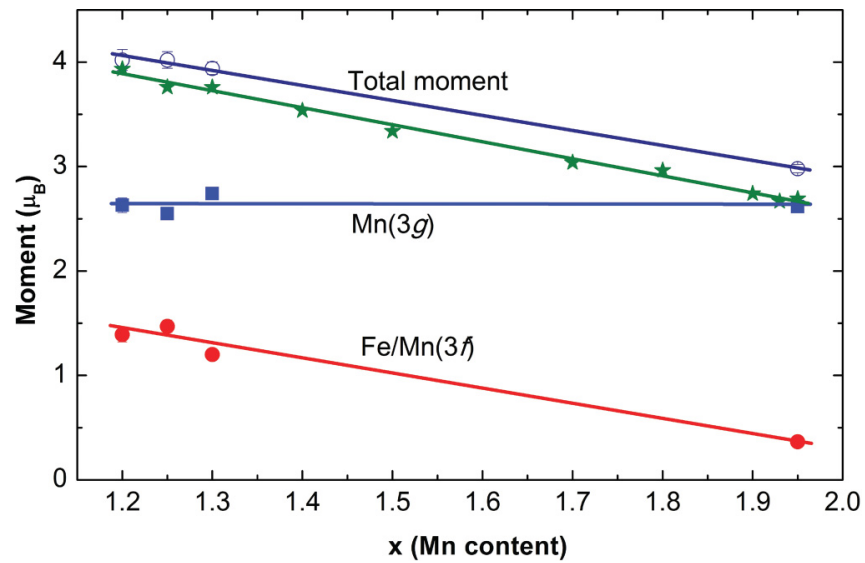

FIG. 3. (Color online) Composition dependence of the magnetic moments derived from the neutron diffraction patterns measured at 3-10 $\mathrm{K}$ for $\mathrm{Mn}_{x} \mathrm{Fe}_{1.95-x} \mathrm{P}_{0.50} \mathrm{Si}_{0.50}$. The total magnetic moments per formula unit (empty circles) are very close to those obtained from saturation magnetization measurements at $5 \mathrm{~K}$ (solid stars). 


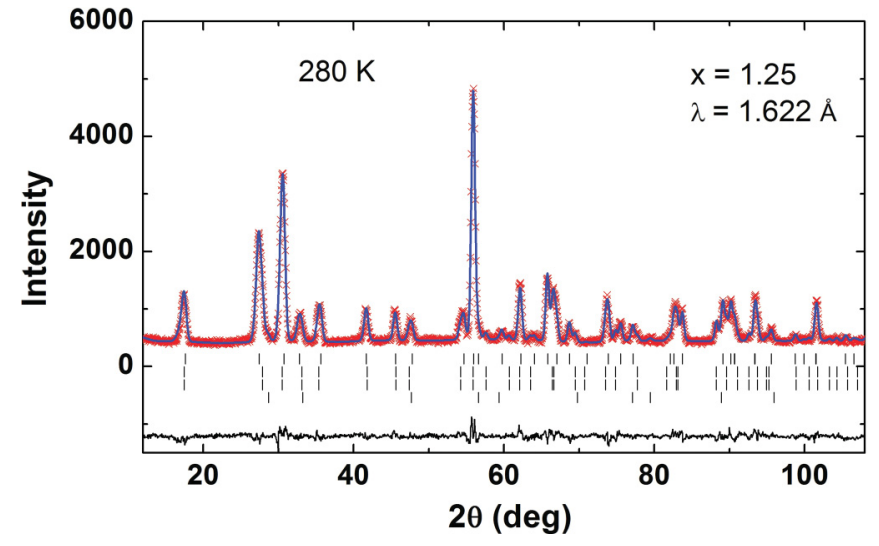

FIG. 4. (Color online) Observed (crosses) and calculated (continuous lines) neutron diffraction patterns for $\mathrm{Mn}_{1.25} \mathrm{Fe}_{0.70} \mathrm{P}_{0.50} \mathrm{Si}_{0.50}$ $(\lambda=1.622 \AA)$ at $280 \mathrm{~K}$ at which the ferro- and paramagnetic phases are coexistent. The lattice parameters and interatomic distances between the magnetic atoms and their nearest neighbors are listed in Table II. From top to bottom, vertical lines indicate the diffraction peaks for the nuclear structure of the paramagnetic phase, the nuclear and magnetic structure of the ferromagnetic phase, and the impurity phase $(\mathrm{Mn}, \mathrm{Fe})_{3} \mathrm{Si}$.

parameter increases with increasing temperature. This happens gradually near and below $T_{\mathrm{C}}$ and abruptly at the transition. The interatomic distances of the ferro- and paramagnetic phases at $T_{\mathrm{C}}$ are listed in Table II. The mean $\mathrm{Mn}(3 g)-\mathrm{P} / \mathrm{Si}$ and $\mathrm{Fe} / \mathrm{Mn}(3 f)-\mathrm{P} / \mathrm{Si}$ distances show almost no change at the transition temperature, while sudden changes in the mean distances between the magnetic atoms are observed. It should be noted that the distances between the intralayer magnetic atoms drop strongly, enhancing the overlap between

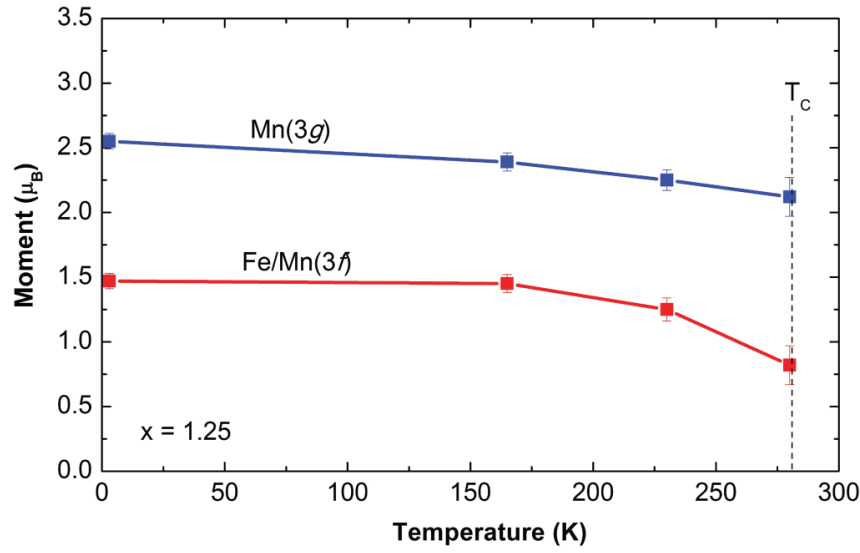

FIG. 5. (Color online) Ordered magnetic moments as a function of temperature derived from neutron diffraction patterns measured at fixed temperatures upon heating for $\mathrm{Mn}_{1.25} \mathrm{Fe}_{0.70} \mathrm{P}_{0.50} \mathrm{Si}_{0.50}$.

the $3 d$ states and resulting in an abrupt reduction of the magnetic moments. However, the relative displacement of the $\operatorname{Mn}(3 g)$ atoms during the transition is smaller than that of the $\mathrm{Fe} / \mathrm{Mn}(3 f)$ atoms. Furthermore, the intralayer $\operatorname{Mn}(3 g)$ $\mathrm{Mn}(3 g)$ distance is larger than the intralayer $\mathrm{Fe} / \mathrm{Mn}(3 f)$ $\mathrm{Fe} / \mathrm{Mn}(3 f)$ distance, and the $\mathrm{Mn} 3 d$ electrons are more localized. Thus, the reduction of the $\operatorname{Mn}(3 g)$ moments is much smaller than that of the $\mathrm{Fe} / \mathrm{Mn}(3 f)$ moments. In terms of mean distances, the reduction of the distances between the intralayer magnetic atoms makes the main contribution to the decrease in the magnetic moments, especially for the $\mathrm{Fe} / \mathrm{Mn}(3 f)$ layers. This may be a common feature for all other hexagonal $\mathrm{Fe}_{2} \mathrm{P}$-based materials exhibiting a first-order magneto-elastic transition. ${ }^{17}$ The above arguments confirm

TABLE II. Lattice parameters and interatomic distances of $\mathrm{Mn}_{1.25} \mathrm{Fe}_{0.70} \mathrm{P}_{0.50} \mathrm{Si}_{0.50}$ at $280 \mathrm{~K}$ at which the ferro- and paramagnetic phases are coexistent. These data are derived from neutron diffraction pattern in Fig. 4.

\begin{tabular}{|c|c|c|c|c|}
\hline & & & \multicolumn{2}{|c|}{$280 \mathrm{~K}$} \\
\hline & & & Ferromagnetic & Paramagnetic \\
\hline \multicolumn{5}{|l|}{ Lattice parameters } \\
\hline$a(\AA)$ & & & $6.1638(1)$ & $6.1163(1)$ \\
\hline$c(\AA)$ & & & $3.3653(1)$ & $3.4212(1)$ \\
\hline$V\left(\AA^{3}\right)$ & & & $110.726(4)$ & $110.837(3)$ \\
\hline \multicolumn{5}{|c|}{ Interatomic distances: $\mathrm{Mn}(3 g)$-nearest neighbors $(\AA)$} \\
\hline $\operatorname{Mn}(3 g)-\operatorname{Mn}(3 g)$ & Intralayer & $\times 4$ & $3.242(6)$ & $3.196(4)$ \\
\hline \multirow[t]{3}{*}{$\operatorname{Mn}(3 g)-\mathrm{Fe} / \mathrm{Mn}(3 f)$} & Interlayer & $\times 1$ & $2.670(7)$ & $2.660(3)$ \\
\hline & Interlayer & $\times 2$ & $2.764(6)$ & $2.790(3)$ \\
\hline & Mean distance & & $2.733(4)$ & $2.747(2)$ \\
\hline \multirow[t]{3}{*}{$\mathrm{Mn}(3 g)-\mathrm{P} / \mathrm{Si}$} & Interlayer & $\times 4$ & $2.517(3)$ & $2.516(3)$ \\
\hline & Intralayer & $\times 1$ & $2.501(6)$ & $2.522(4)$ \\
\hline & Mean distance & & $2.514(2)$ & $2.517(1)$ \\
\hline \multicolumn{5}{|c|}{ Interatomic distances: $\mathrm{Fe} / \mathrm{Mn}(3 f)$-nearest neighbors $(\AA)$} \\
\hline $\mathrm{Fe} / \mathrm{Mn}(3 f)-\mathrm{Fe} / \mathrm{Mn}(3 f)$ & Intralayer & $\times 2$ & $2.753(6)$ & $2.696(3)$ \\
\hline \multirow[t]{3}{*}{$\mathrm{Fe} / \mathrm{Mn}(3 f)-\mathrm{Mn}(3 g)$} & Interlayer & $\times 1$ & $2.670(7)$ & $2.660(3)$ \\
\hline & Interlayer & $\times 2$ & $2.764(6)$ & $2.790(3)$ \\
\hline & Mean distance & & $2.733(4)$ & $2.747(2)$ \\
\hline \multirow[t]{3}{*}{$\mathrm{Fe} / \mathrm{Mn}(3 f)-\mathrm{P} / \mathrm{Si}$} & Intralayer & $\times 2$ & $2.322(3)$ & $2.318(2)$ \\
\hline & Interlayer & $\times 2$ & $2.315(3)$ & $2.313(2)$ \\
\hline & Mean distance & & $2.319(2)$ & $2.316(1)$ \\
\hline
\end{tabular}



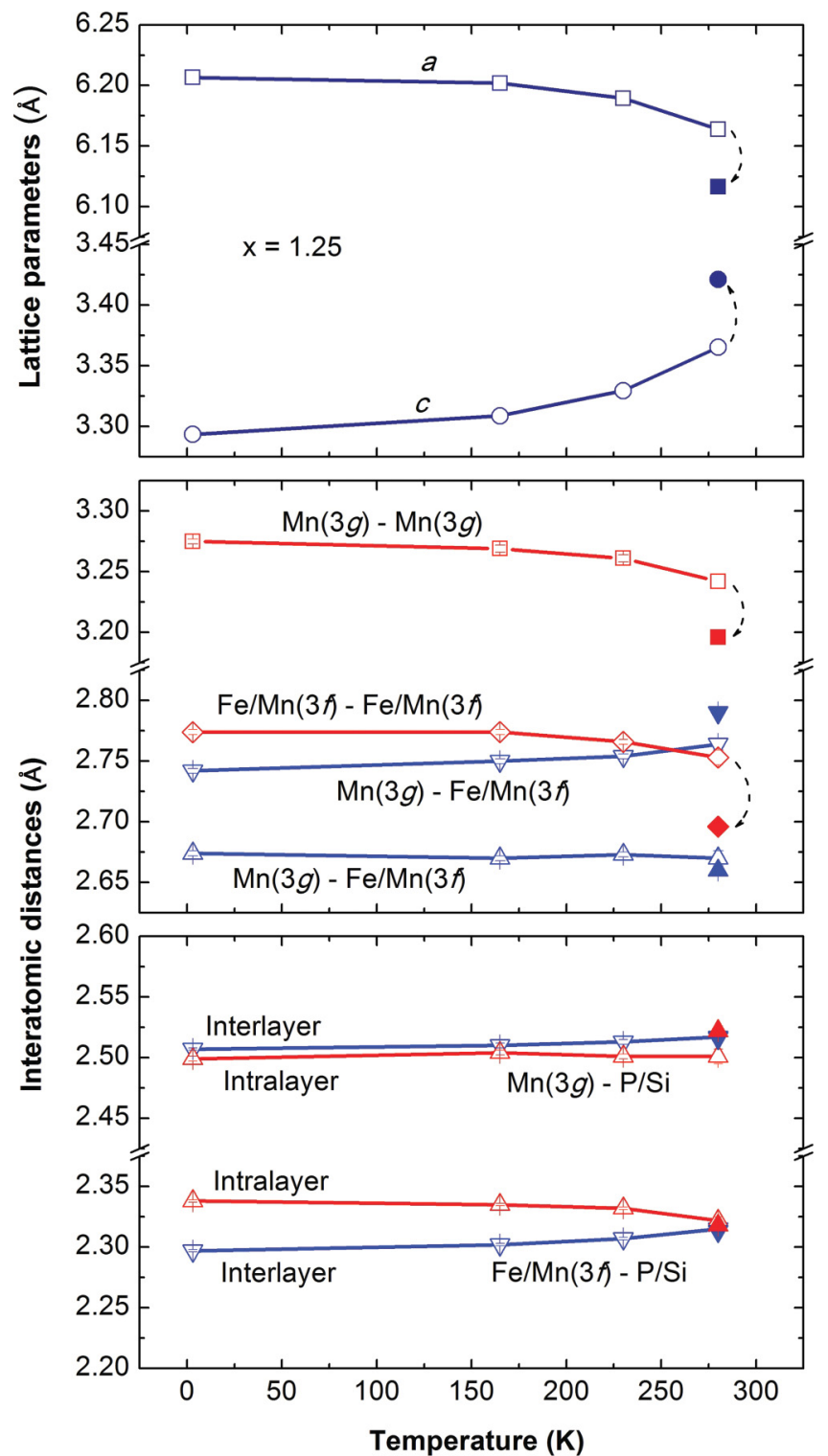

FIG. 6. (Color online) Lattice parameters and interatomic distances as a function of temperature derived from neutron diffraction patterns measured at fixed temperatures upon heating for $\mathrm{Mn}_{1.25} \mathrm{Fe}_{0.70} \mathrm{P}_{0.50} \mathrm{Si}_{0.50}$ in the ferromagnetic (empty symbols) and paramagnetic (solid symbols) state.

our proposal for the competition between chemical bonding and moments. The observed behaviors of the moments and the lattice parameter below $T_{\mathrm{C}}$ indicate that the competition between chemical bonding and moments becomes stronger when $T_{\mathrm{C}}$ is approached.

The value of the lattice parameters obtained from the neutron diffraction measurements is in good agreement with that derived from $x$-ray diffraction patterns by using the FullProf refinement program (see Fig. 7). It should be noted that, above $T_{\mathrm{C}}$, we observe nonlinear temperature variations of the lattice parameters up to a certain temperature $T_{\mathrm{O}}$, while the volume increases linearly with temperature. It seems that the competition between chemical bonding and moments does not affect the volume because there is only a small volume change of about $0.1 \%$ at $T_{\mathrm{C}}$, and a linear variation of the volume

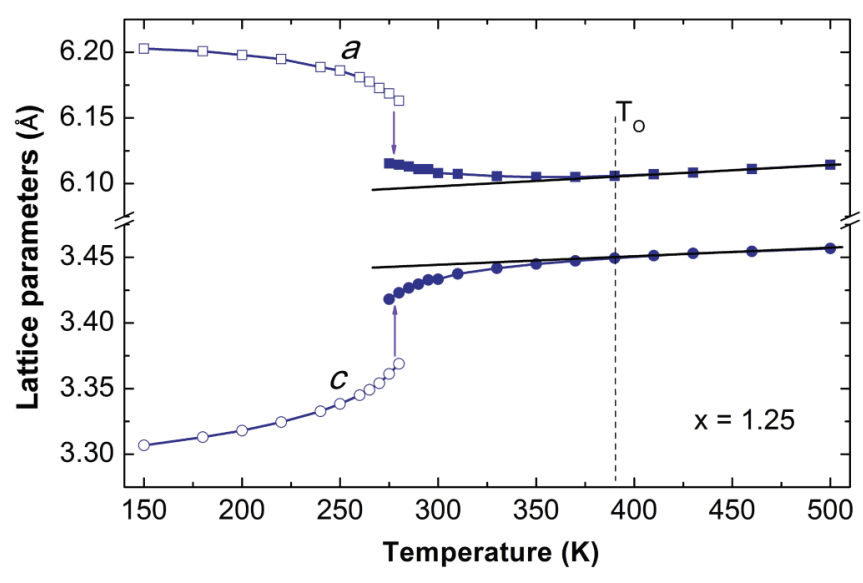

FIG. 7. (Color online) Lattice parameters as a function of temperature derived from $\mathrm{x}$-ray diffraction patterns measured at fixed temperatures upon heating for $\mathrm{Mn}_{1.25} \mathrm{Fe}_{0.70} \mathrm{P}_{0.50} \mathrm{Si}_{0.50}$. The error bar is smaller than the size of the symbols.

is also observed at temperatures well below $T_{\mathrm{C}}$. The value of $T_{\mathrm{O}}$ is determined to be about $390 \mathrm{~K}$ for the $x=1.25$ sample. The nonlinear variations of the lattice parameters imply that the competition between chemical bonding and moments still continues above $T_{\mathrm{C}}$. Thus, for the Mn-rich compounds, the $\mathrm{Fe} / \mathrm{Mn}(3 f)$ moments do not actually disappear at $T_{\mathrm{C}}$, but their size significantly drops to a lower value. With further increasing temperature, the $\mathrm{Fe} / \mathrm{Mn}(3 f)$ moments gradually decrease and vanish at $T_{\mathrm{O}}$, while the $\mathrm{Mn}(3 g)$ moments prevail above $T_{\mathrm{O}}$. The evolution of these moments leads to the nonlinear variations of the lattice parameters via the magnetoelastic coupling. Thus, $T_{\mathrm{O}}$ represents the onset of the moment formation on the $3 f$ site.

In Fig. 8, the magnetic susceptibility at temperatures above $T_{\mathrm{C}}$ is shown for the $x=1.25$ sample. In the paramagnetic state, the magnetic susceptibility deviates from the Curie-Weiss law, up to $T^{*} \sim 630 \mathrm{~K}$. The paramagnetic Curie temperature $\left(\theta_{\mathrm{p}}\right)$ is estimated to be about $400 \mathrm{~K}$. The deviation of the susceptibility from the Curie-Weiss law is a general feature for hexagonal $\mathrm{Fe}_{2} \mathrm{P}$-based compounds and is often ascribed to short-range magnetic order. ${ }^{18-20}$ We suggest that the decrease

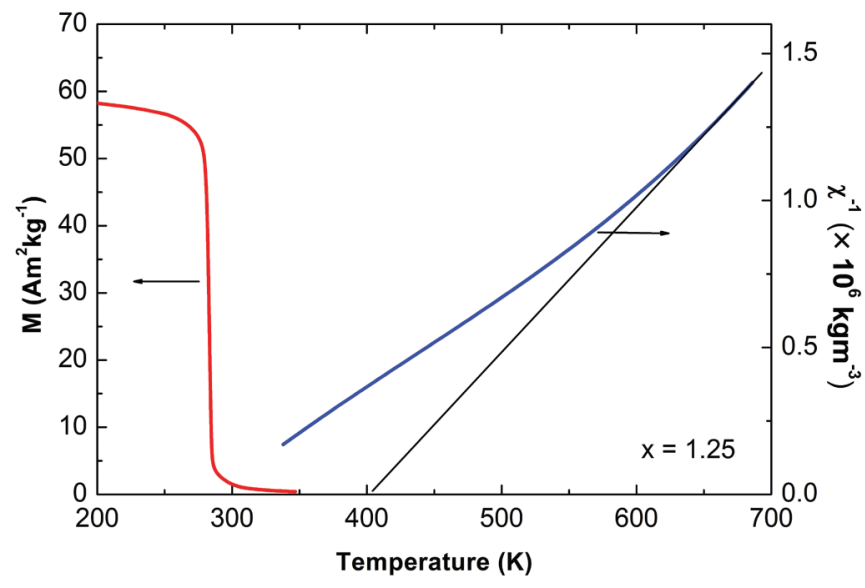

FIG. 8. (Color online) Magnetization and inverse susceptibility as a function of temperature measured in $0.1 \mathrm{~T}$ upon heating for $\mathrm{Mn}_{1.25} \mathrm{Fe}_{0.70} \mathrm{P}_{0.50} \mathrm{Si}_{0.50}$. 
in the magnetic moments upon heating also contributes to the nonlinear variation of the inverse susceptibility at temperatures below $T_{\mathrm{O}}$. Using the Curie-Weiss law, the effective moment per formula unit ( $\mu_{\text {eff }}$ ) can be derived from the Curie constant (C) by:

$$
C=\frac{\mu_{0} N \mu_{\mathrm{eff}}^{2}}{3 k_{\mathrm{B}}},
$$

where $C$ is taken as inverse slope of the $\chi^{-1}-T$ curve, $N$ is the number of formula units per mass unit, $\mu_{0}$ is the magnetic constant, and $k_{\mathrm{B}}$ is the Boltzmann's constant. The Curie-Weiss fit above $630 \mathrm{~K}$ leads to a $\mu_{\mathrm{eff}}$ of about $4.2 \mu_{\mathrm{B}}$ for the $x=1.25$ sample.

The Rhodes-Wohlfarth ratio $q_{\mathrm{c}} / q_{\mathrm{s}}{ }^{21,22}$ is frequently used to distinguish between local moment and itinerant-electron ferromagnetism. Here, $q_{\mathrm{c}}$ and $q_{\mathrm{s}}$ are deduced from the Curie constant and from the low-temperature saturation magnetization, respectively, by using the formulas:

$$
\mu_{\mathrm{eff}}^{2}=N_{\mathrm{at}} q_{\mathrm{c}}\left(q_{\mathrm{c}}+2\right) \mu_{\mathrm{B}}^{2}
$$

and

$$
\mu_{\mathrm{s}}=q_{\mathrm{s}} \mu_{\mathrm{B}},
$$

where $N_{\text {at }}$ is the number of magnetic atoms per formula unit and $\mu_{\mathrm{s}}$ is the average ordered moment per magnetic atom at low temperature. For the $x=1.25$ sample, the magnetization data and neutron diffraction measurements at $5 \mathrm{~K}$ give values for $\mu_{\mathrm{s}}$ of 1.9 and $2.1 \mu_{\mathrm{B}}$, respectively. Assuming magnetic moments on the $3 g$ and $3 f$ sites, we obtain a $q_{\mathrm{c}}$ of 2.1 from Eq. (2). The ratio $q_{\mathrm{c}} / q_{\mathrm{s}}$ of about 1 seems to give an indication of localized magnetism. ${ }^{21,22}$ Our calculation for $\mathrm{MnFeP}_{0.85} \mathrm{Ge}_{0.15}$ using the data reported by Yabuta et al. ${ }^{20}$ also leads to the ratio $q_{\mathrm{c}} / q_{\mathrm{s}}$ of 1 . These results are in contradiction with the generally agreed description of the hexagonal $\mathrm{Fe}_{2} \mathrm{P}$-based alloys as itinerantelectron magnetic compounds. We propose that this deviation from the Rhodes-Wohlfarth plot may arise from the large difference between the high- and low-moment phases at low and high temperature, respectively (see Fig. 9).

The low-moment phase, which has only magnetic moments on the $3 g$ sites, only exists in the paramagnetic state at high temperature. It would become ferromagnetic at the parato ferromagnetic transition temperature of the low-moment phase $\left(T_{\mathrm{C}}^{\mathrm{LM}}\right)$ in the vicinity of $\theta_{\mathrm{p}}$. However, the ferromagnetic state of the low-moment phase is apparently not energetically

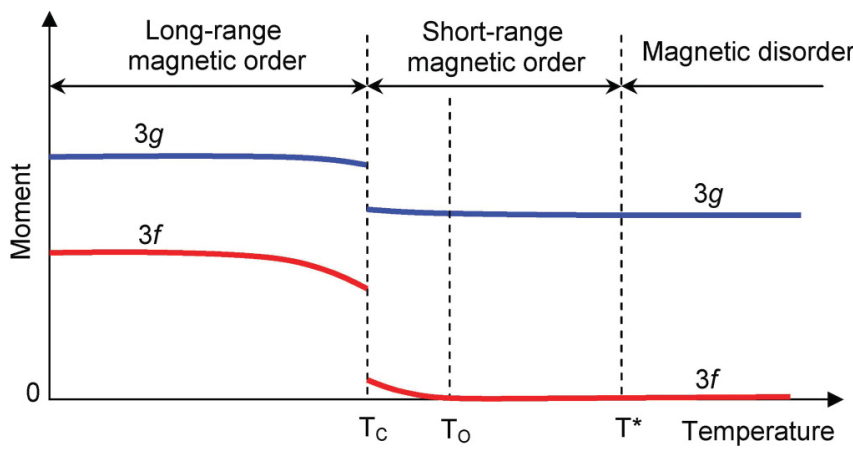

FIG. 9. (Color online) Sketch of the magnetic moments near the first-order magneto-elastic transition in hexagonal Mn-Fe-P-Si compounds. The itinerant moments are not to scale. preferred, which indicates that the high-moment phase possesses a lower free energy under the same condition. From the Rhodes-Wohlfarth plot, ${ }^{21} q_{\mathrm{c}} / q_{\mathrm{s}}$ of an itinerant-electron ferromagnetic system with $T_{\mathrm{C}}=400 \mathrm{~K}$ is roughly estimated to be about 1.7. For the $x=1.25$ sample, since the low-moment phase has $T_{\mathrm{C}}^{\mathrm{LM}}$ somewhat lower than $400 \mathrm{~K}$, we may assume that the ratio $q_{\mathrm{c}} / q_{\mathrm{s}}$ of the low-moment phase is 1.7 . On the other hand, when we assume for the low-moment phase only magnetic moments on the $3 g$ sites, $q_{\mathrm{c}}$ is found to be about 3.3. We therefore deduce $q_{\mathrm{s}} \sim 1.9$, and $\mu_{\mathrm{s}} \sim 1.9 \mu_{\mathrm{B}}$. This value of $\mu_{\mathrm{s}}$ reflects the magnitude of the ordered $\operatorname{Mn}(3 g)$ moments of the low-moment phase at low temperature. Hence, the first-order magneto-elastic transition for the $x=1.25$ sample produces the $\mathrm{Fe} / \mathrm{Mn}(3 f)$ moments of $1.5 \mu_{\mathrm{B}}$ and the $\mathrm{Mn}(3 g)$ moments of $2.6 \mu_{\mathrm{B}}$.

The following physical picture evolves from our results: At high temperatures above $T_{\mathrm{O}}$, Mn on the $3 g$ sites carries magnetic moments, and the $3 f$ sites are predominantly in the bonding state. At lower temperatures, due to ferromagnetic exchange interaction between the $\operatorname{Mn}(3 g)$ moments the $\mathrm{Fe} / \mathrm{Mn}(3 f)$ moments experience a local field that supports the nonbonding high-moment state. Furthermore, it can be seen that magnetic order is enhanced upon cooling. The high-temperature magnetic disorder at $T>T^{*}$ changes into a short-range magnetic order in the range of $T^{*}>T>T_{\mathrm{C}}$ before developing into a long-range magnetic order at $T<$ $T_{\mathrm{C}}$ (see Fig. 9). The enhanced magnetic order which gives rise to an increase in the exchange field therefore supports the formation and evolution of the $\mathrm{Fe} / \mathrm{Mn}(3 f)$ moments. The exchange fields are critical for the moment formation and for the development of ferromagnetic order which occur at $T_{\mathrm{O}}$ and $T_{\mathrm{C}}$, respectively. The short-range magnetic order therefore plays an important role in the mechanism of the firstorder magneto-elastic transition in the hexagonal Mn-Fe-P-Si compounds. An external magnetic field can also support the nonbonding high-moment state and make the transition happen at higher temperatures (see Fig. 10). The value of $d T_{\mathrm{C}} / d B$ is estimated to be about $3.0-3.5 \mathrm{~K} / \mathrm{T}$ for the $x=1.25$ sample.

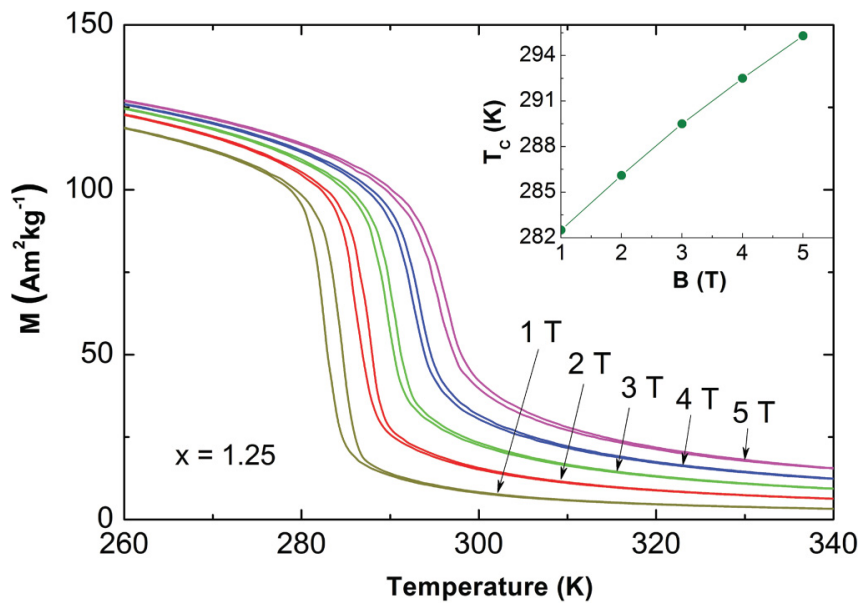

FIG. 10. (Color online) Temperature dependence of the magnetization measured in different magnetic fields (from 1 to $5 \mathrm{~T}$ ) upon cooling and heating for $\mathrm{Mn}_{1.25} \mathrm{Fe}_{0.70} \mathrm{P}_{0.50} \mathrm{Si}_{0.50}$. The inset shows the field dependence of the transition temperature derived from the $M-T$ curves measured upon cooling. 


\section{CONCLUSIONS}

In summary, a general physical picture of the evolution of the magnetic moments has been put forward for the Mn-rich hexagonal Mn-Fe-P-Si compounds with first-order magneto-elastic transition. The substitution of $\mathrm{Mn}$ for $\mathrm{Fe}$ on the $3 f$ sites does not cause a change in the $\operatorname{Mn}(3 g)$ magnetic moments but results in a linear decrease in the magnitude of the $\mathrm{Fe} / \mathrm{Mn}(3 f)$ magnetic moments. The reduction of the magnetic moments with increasing temperature below the transition temperature has been observed by using neutron diffraction. The sudden change in the interatomic distances validates our description of high/low-moment phase transition, which leads to the deviation from the Rhodes-Wohlfarth plot. The formation and gradual development of the magnetic moment on the $3 f$ sites upon cooling give rise to anomalous thermal expansion in the paramagnetic state. By exploiting high-temperature magnetic-susceptibility measurement, we demonstrate the difference of the magnitude of the magnetic moments at low and high temperatures. This paper also reveals the role of short-range magnetic order in the development of the first-order magneto-elastic transition. These results support our proposal that the competition between the moments and chemical bonding is at the core of giant MCE displayed in the class of hexagonal $\mathrm{Fe}_{2} \mathrm{P}$-based materials with first-order magneto-elastic transition.

\section{ACKNOWLEDGMENTS}

The authors would like to thank Anton J. E. Lefering, Michel P. Steenvoorde, and Bert Zwart (Delft University of Technology) for their help in magnetic and structural measurements and sample preparation. This work is part of the Industrial Partnership Program I18 of the Dutch Foundation for Fundamental Research on Matter (FOM), and cofinanced by BASF Future Business.
*Corresponding author: h.d.nguyen@tudelft.nl

${ }^{1}$ E. Brück, J. Phys. D Appl. Phys. 38, R381 (2005).

${ }^{2}$ V. K. Pecharsky and K. A. Gschneidner, Phys. Rev. Lett. 78, 4494 (1997).

${ }^{3}$ A. Fujita, S. Fujieda, Y. Hasegawa, and K. Fukamichi, Phys. Rev. B 67, 104416 (2003).

${ }^{4}$ N. T. Trung, L. Zhang, L. Caron, K. H. J. Buschow, and E. Brück, Appl. Phys. Lett. 96, 172504 (2010).

${ }^{5}$ T. Krenke, E. Duman, M. Acet, E. F. Wassermann, X. Moya, L. Manosa, and A. Planes, Nat. Mater. 4, 450 (2005).

${ }^{6}$ O. Tegus, E. Brück, K. H. J. Buschow, and F. R. de Boer, Nature 415, 150 (2002).

${ }^{7}$ N. H. Dung, Z. Q. Ou, L. Caron, L. Zhang, D. T. C. Thanh, G. A. de Wijs, R. A. de Groot, K. H. J. Buschow, and E. Brück, Adv. Energy Mater. 1, 1215 (2011).

${ }^{8}$ D. Haskel, Y. B. Lee, B. N. Harmon, Z. Islam, J. C. Lang, G. Srajer, Y. Mudryk, K. A. Gschneidner, and V. K. Pecharsky, Phys. Rev. Lett. 98, 247205 (2007).

${ }^{9}$ M. D. Kuz'min and M. Richter, Phys. Rev. B 76, 092401 (2007).

${ }^{10}$ J. Lyubina, K. Nenkov, L. Schultz, and O. Gutfleisch, Phys. Rev. Lett. 101, 177203 (2008).
${ }^{11}$ N. H. Dung, L. Zhang, Z. Q. Ou, and E. Brück, Appl. Phys. Lett. 99, 092511 (2011).

${ }^{12}$ K. D. Liss, B. Hunter, M. Hagen, T. Noakes, and S. Kennedy, Physica B 385-386, 1010 (2006).

${ }^{13}$ A. W. Hewat, Mater. Sci. Forum 9, 69 (1986).

${ }^{14}$ J. Rodriguez-Carvajal, Physica B 192, 55 (1993).

${ }^{15}$ V. Hoglin, M. Hudl, M. Sahlberg, P. Nordblad, P. Beran, and Y. Andersson, J. Solid State Chem. 184, 2434 (2011).

${ }^{16}$ L. Zhang, O. Moze, K. Prokes, O. Tegus, and E. Brück, J. Magn. Magn. Mater. 290, 679 (2005).

${ }^{17}$ D. M. Liu, Q. Z. Huang, M. Yue, J. W. Lynn, L. J. Liu, Y. Chen, Z. H. Wu, and J. X. Zhang, Phys. Rev. B 80, 174415 (2009).

${ }^{18}$ O. Beckman and L. Lundgren, in Handbook of Magnetic Materials, edited by K. H. J. Buschow (Elsevier, Amsterdam, 1991), Vol. 6, pp. 181.

${ }^{19}$ R. Zach, M. Guillot, and R. Fruchart, J. Magn. Magn. Mater. 89, 221 (1990).

${ }^{20}$ H. Yabuta, K. Umeo, T. Takabatake, K. Koyama, and K. Watanabe, J. Phys. Soc. Jpn. 75, 113707 (2006).

${ }^{21}$ P. Rhodes and E. P. Wohlfarth, Proc. R. Soc. A 273, 247 (1963).

${ }^{22}$ E. P. Wohlfarth, J. Magn. Magn. Mater. 7, 113 (1978). 